\title{
Special Shielded Resistor for High-Voltage D-C Measurements
}

\author{
J. H. Park
}

(September 26, 1961)

\begin{abstract}
A new design for an accurate high-voltage d-c standard resistor has been devised. It is made up of a large number of individually shielded, one-megohm wire-wound resistors connected in series and arranged to form a vertical helix between a ground plate and a highvoltage electrode. The individual shields completely enclose each one-megohm resistor and prevent formation of corona at the surface of the resistance coil no matter how high the potential of the shield is above ground. The vertical helical configuration with a large "hat," or high-voltage electrode, on top serves to prevent concentration of electric field and corona formation at the high-potential end of the resistor. A 200-megohm unit, constructed during 1955 and tried out up to $100 \mathrm{kv}$ in 1956 , indicated the design to be free of corona errors but, for the particular one-megohm resistors used, the variation with temperature was quite large $\left(0.01\right.$ percent per $\left.{ }^{\circ} \mathrm{C}\right)$. A 100 megohm unit using low-temperature coefficient resistors has been recently constructed and tested up to $100 \mathrm{kv}$.

An experimental method of checking for corona or leakage errors at high values of voltage was developed. It consisted of accurately comparing the current "in" at the high voltage end of the resistor with the current "out" at the ground end for several different values of applied voltage. These measurements together with others performed to check temperature and leakage errors indicated that the value of resistance for the 100-megohm unit remains constant to within about $10 \mathrm{ppm}$ for voltages up to $50 \mathrm{kv}$ under ordinary laboratory conditions. At $100 \mathrm{kv}$ the maximum error (caused by heating) was estimated to be about $40 \mathrm{ppm}$.
\end{abstract}

\section{Introduction}

Accurate measurements of $d$-c voltages at values above $10 \mathrm{v}$ are nearly always dependent upon a resistive voltage divider which consists of a high resistance, $R_{1}$, in series with a low resistance, $R_{2}$. The voltage to be measured is connected across the series combination with $R_{2}$ at the ground end. The divider ratio, $\left(R_{1}+R_{2}\right) / R_{2}$, is chosen to give about a 1 v drop across $R_{2}$, which can then be measured with a null potentiometer. This is the well known "volt-box" method and tapped resistors or "volt boxes" are readily available for voltages up to at least $1,500 \mathrm{v}$. In extending this method to higher voltages the only additional difficulty is in designing a high-voltage resistor with an effective value which does not change with voltage.

A change in effective resistance with voltage may be due to any one or a combination of three factors: (1) heating of the resistance wire due to the $I^{2} R$ loss - the magnitude of this change will depend upon the temperature coefficient of the entire resistor; (2) current leakage through the volume or over the surface of the insulation used to support the resistor - such leakage usually increases with voltage and in effect decreases the total resistance; (3) corona discharges which may appear at locations of high gradient along the resistor as the voltage is increased-in effect they "leak" part of the resistor current to ground. The heating effect, factor (1) can be quite bothersome unless the overall temperature coefficient is very low. However, a highvoltage resistor is most conveniently made up by connecting a large number of one-megohm units in series. Thus, temperature coefficient can be reduced to a negligible minimum by choosing some resistors with positive and others with negative temperature coefficients. This can readily be done if a large number of one-megohm units made up of either Karma ${ }^{1}$ or Evanohm ${ }^{1}$ wire are available and their temperature coefficients have been measured. Factors (2) and (3), above, cannot be measured and reduced to a low magnitude in any such simple manner. Thus, the primary design problem is to keep leakage and corona effects as low as possible. Also, some experimental method must be devised to check for these effects with full voltage applied to the complete resistor.

1 Trade names for special alloys $(\mathrm{NiCr}+\mathrm{Al}+\mathrm{Fe})$ having high resistivity and low temperature coefficient of resistance. 


\section{Helical Resistor Made in 1955}

A special helical design to keep corona effects to a minimum when a large number of 1-megohm resistors are connected in series was first devised in January 1955. It was made up of 200 one-megohm wirewound resistor units which had been obtained on surplus after World War II. Each unit consisted of wire wound on a cylindrical spool $3 / 4 \mathrm{in}$. in diameter and $1 \frac{1}{4} \mathrm{in}$. long and connected to screw terminals at each end of the cylinder. Overlapping brass caps with ribbon insulation between them were attached to the screw terminals as indicated in figure 1 . These two brass caps form a complete potential shield around the unit and definitely fix the voltage gradient environment immediately surrounding the resistance wire. Such voltage gradients are determined only by the potential difference between the two caps which is equal to the $I R$ drop across the resistor. Thus the possibility of corona appearing in the air immediately surrounding the resistance wire is eliminated no matter how high the potential of the resistor and its shields may be with respect to ground.

A jig was made to support the individually shielded units along a circular helical path. They were then soldered together in this position and mounted on a lucite tube $4 \mathrm{in}$. in diameter and $39 \mathrm{in}$. high as shown in figure 2. The circular helical shape keeps a uniform potential drop per turn along the lucite tube. The 24 inch diameter copper "hat" placed on top of the lucite tube serves to intercept electrostatic lines of force from objects at ground potential and prevents concentration of such lines of force at the top turns of the resistor helix. Thus the gradient at the outside surface of the individual resistor caps is very nearly the same for units near the top as those near the bottom, and the possibility of corona from these shield caps is greatly reduced or eliminated.

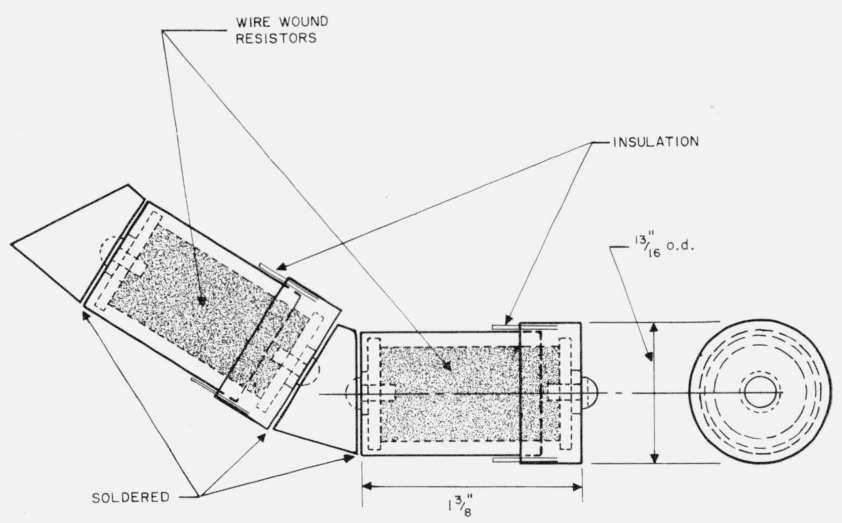

Figure 1. Two one-megohm wire-wound resistors, mounted inside their shield caps, as used in first model of helical resistor.

Indicates method of connecting resistors in series to form helix.

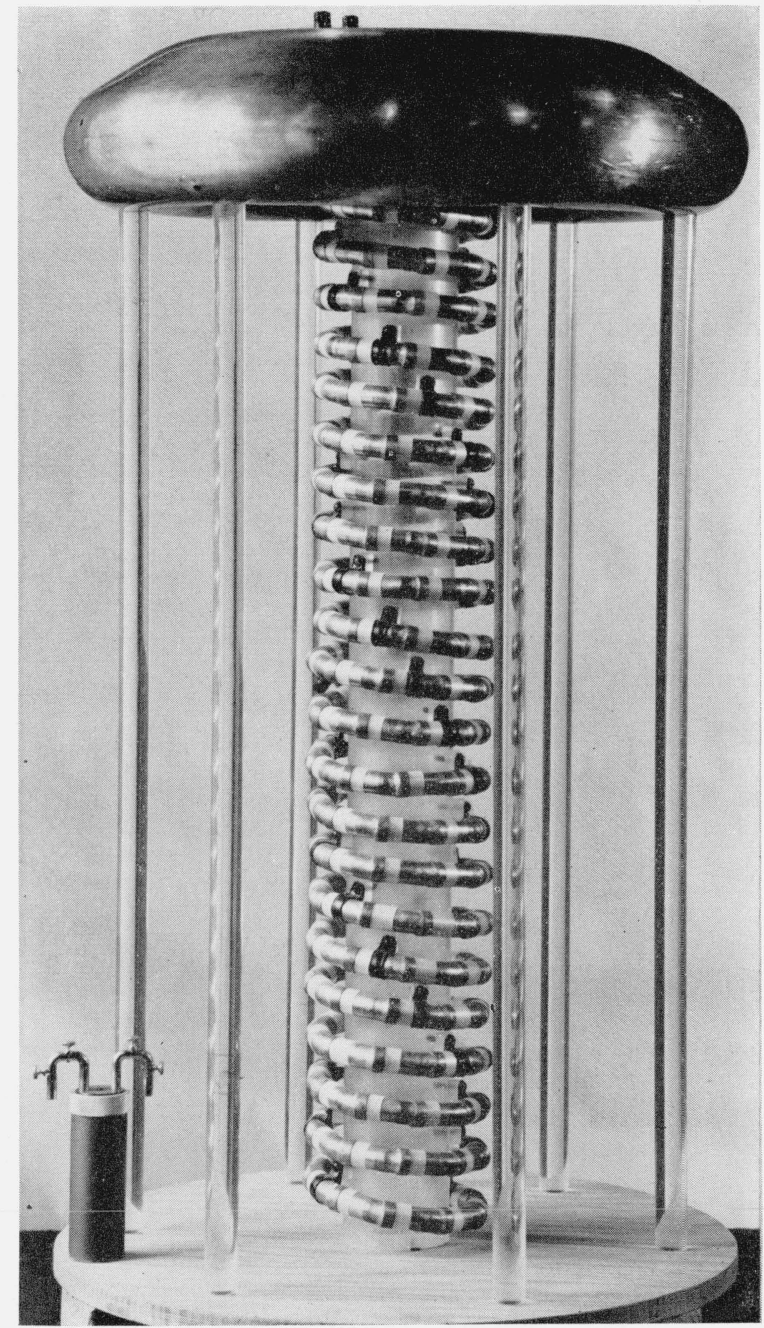

FIGURE 2. The first model of helical resistor.

Made during 1955.

If there were any corona discharge or even "dark current" from the resistor at some point between the high-voltage electrode and the low-voltage lead, it would cause the current "out" at the low-voltage lead to be less than the current "in" at the highvoltage electrode. This would introduce a change in the value of effective resistance which is used in voltage measurements. Current leakage through or over the surface of any insulating supports would cause a similar type of error. An experimental check for such errors can be obtained by getting. a simultaneous measurement on the current "in" and "out" at various voltages up to the highest which can be used. This was done by adjusting the applied voltage to give a certain fixed value of current "in" under steady conditions and measuring the current "out".

The current "in" was set to a fixed known value by 
passing it through a standard resistor and balancing the IR drop to that of a standard cell by use of a reflecting mirror galvanometer. The standard resistor, standard cell, and galvanometer were placed on top of the high-voltage electrode and covered with a 3-gal cylindrical can to prevent corona discharges from them. The resistance of the standard resistor used in this circuit was chosen to give the value of current desired. The current "out" was measured in the usual manner by passing it through a standard resistor and measuring the IR drop with a null potentiometer. The procedure then was to choose a value of standard resistor to give the required current "in" and adjust this current to give a balance on the reflecting galvanometer mounted inside the 3-gal can. When a balance was attained the current "out" was read on the potentiometer at the ground end. Any discrepancy between these two currents indicated either a leakage or corona effect.

Some special precautions taken during these measurements should be noted. The switch closing the galvanometer circuit inside the 3 -gal can must not be closed until the current has been approximately adjusted to the correct value. This requires a remotely operated switch because the 3 -gal can is at high voltage. A waxed cord was tried first for operating this switch but found to be unsatisfactory because it jarred the galvanometer off scale. A relay operated by a photoelectric cell circuit which could be closed by a beam of light was installed inside the 3-gal can and found to operate satisfactorily. It was also found desirable to place extra filter capacitors across the high-voltage supply, giving high $\mathrm{RC}$ time constants on both charge and discharge, thus serving to keep the current steady.

Current measurements were made using 5,000- and $2,000-\mathrm{ohm}$ standard resistors in the 3 -gal can giving voltage values of approximately 40 and $100 \mathrm{kv}$ across the 200-megohm resistor. At $40 \mathrm{kv}$ the current "in" and current "out" agreed to within about $20 \mathrm{ppm}$ which was considered the limit of accuracy for this particular test. At $100 \mathrm{kv}$ the agreement could be certain only to within about $70 \mathrm{ppm}$ because of unsteadiness in the supply voltage. No further attempt to minimize these measurement errors was made at that time because the expected accuracy of the first model was about \pm 0.01 percent.

Actually the largest error in this model was found to be due to the temperature coefficient of resistance of the 1-megohm resistors. Apparently Nichrome V wire had been used in winding these resistors. The measured change of resistance with room temperature as determined with low voltage applied was +0.01 percent per degree $\mathrm{C}$ increase in ambient temperature. The increase of resistance with applied voltage followed the square law as expected, and this total change from low to rated voltage of $100 \mathrm{kv}$ was 0.09 percent. Correction curves were derived for changes in applied voltage and room temperature. It was concluded that, with care in applying these corrections, accuracies within 0.02 percent could be expected for applied voltage up to $50 \mathrm{kv}$.

\section{Improved Helical Resistor Built in 1961}

The first model has been used as a standard for calibrating other high-voltage resistors at the National Bureau of Standards for several years. In most cases the accuracy attainable was sufficient, but in several instances accuracy needs reached 0.01 percent or better. In view of the ever higher accuracy needs as time goes on, especially in experiments involving the accurate measurement of atomic and nuclear constants, it was decided to build another resistor of the same design using 1-megohm units with lower temperature coefficients.

In 1960 it was found that due to improvements in resistor winding techniques and to the development of special alloy wire of low temperature coefficient, it was possible to obtain 1-megohm units guaranteed to have a temperature coefficient less than $5 \mathrm{ppm}$ per degree C. A large number were ordered, and on delivery their temperature coefficients were determined by measurements at three or more temperatures between 20 and $44^{\circ} \mathrm{C}$. They were all well within the $5 \mathrm{ppm}$ per degree C limit-also some resistors increased and others decreased with rising temperature and in general the change was proportional to temperature. Thus it was possible to choose matched pairs of these resistors with nearly zero average temperature coefficient. By connecting such matched pairs in series, a high-voltage resistor with nearly zero change with temperature was made.

To keep corona and leakage errors as low as possible the same spiral type of construction as that in the first model was used. A new technique for making the shields and connecting them in a helix was devised. Each shielded unit contained two resistors and was assembled from brass parts, machined out of tubing, and a $45^{\circ}$ brass elbow, as shown in figure 3. Polyethylene sheet insulation was used between the overlapping shields around each resistor as before. Eight of these units, fitted together, constitute a single turn of the helix with an outside diameter of $9 \frac{1}{2}$ in. They were fastened to a 7 -in.diam lucite tube ( $1 / 8$ in. wall thickness) using one nylon clamp and screw for each unit. The pitch of the helix was chosen to prevent any possibility of corona between adjacent turns.

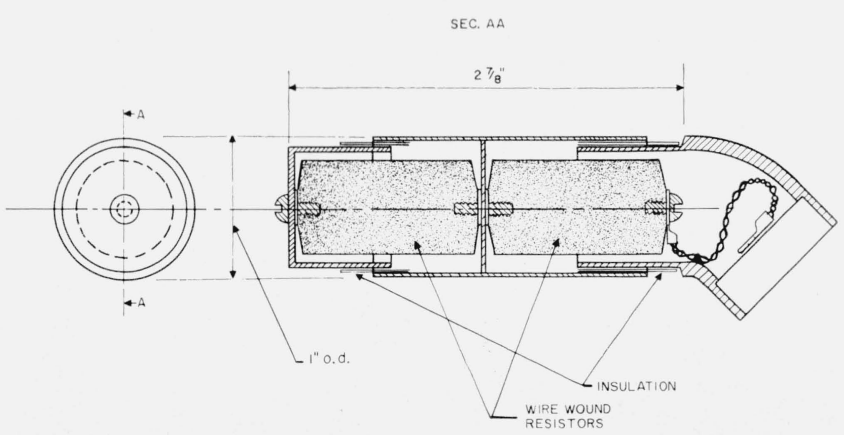

FIgURE 3. A two-resistor shielded unit as used in the second model of helical resistor. 
First an estimate of required pitch was made based on a maximum rated voltage for each 1-megohm resistor of $1,000 \mathrm{v}$. This gave 16 kilovolts per turn. A pitch of $2 \frac{3}{8}$ in. per turn was taken, giving $13 / 8$ in. between adjacent brass shields from one turn to the next. A $1 \frac{1}{2}$-turn resistor (maximum rating $24 \mathrm{kv}$ ) was made up using this pitch and its resistance was measured at various voltages up to $27 \mathrm{kv}$. This was done using a wheatstone bridge with the 1955 model 200-megohm helical resistor as the other highvoltage arm. Only gradual uniform changes in resistance were noted as voltage was increased in steps from 5 to $27 \mathrm{kv}$, and they could be accounted for by heating effects. There was no evidence of corona effects even at $27 \mathrm{kv}$, which is 125 percent of the maximum to be used.

On the basis of these preliminary tests a 100megohm resistor of $6 \frac{1}{4}$ turns was made up with the same pitch. The lucite tube is $16 \frac{1}{2}$ in. long and mounted vertically between a brass ground plate and a high-voltage electrode as shown by the photograph in figure 4. The electrode on top of the lucite tube was designed so that another similar resistor could be placed on top of it. By stacking resistors and connecting them in series, voltage ranges in multiples of $100 \mathrm{kv}$ could be obtained. At present only one 100megohm resistor has been constructed because so far there has been no urgent requirement for very accurate measurements at voltages above $50 \mathrm{kv}$. However, corona tests on the high-voltage electrode were made up to $200 \mathrm{kv}$ by using two $100 \mathrm{kv}$ power supplies. The normally grounded brass plate was supported on ceramic insulators and connected to the minus $100 \mathrm{kv}$. The high-voltage electrode was connected to the plus $100 \mathrm{kv}$. For this test the 100 1-megohm resistors were removed but the same lucite tube was used to

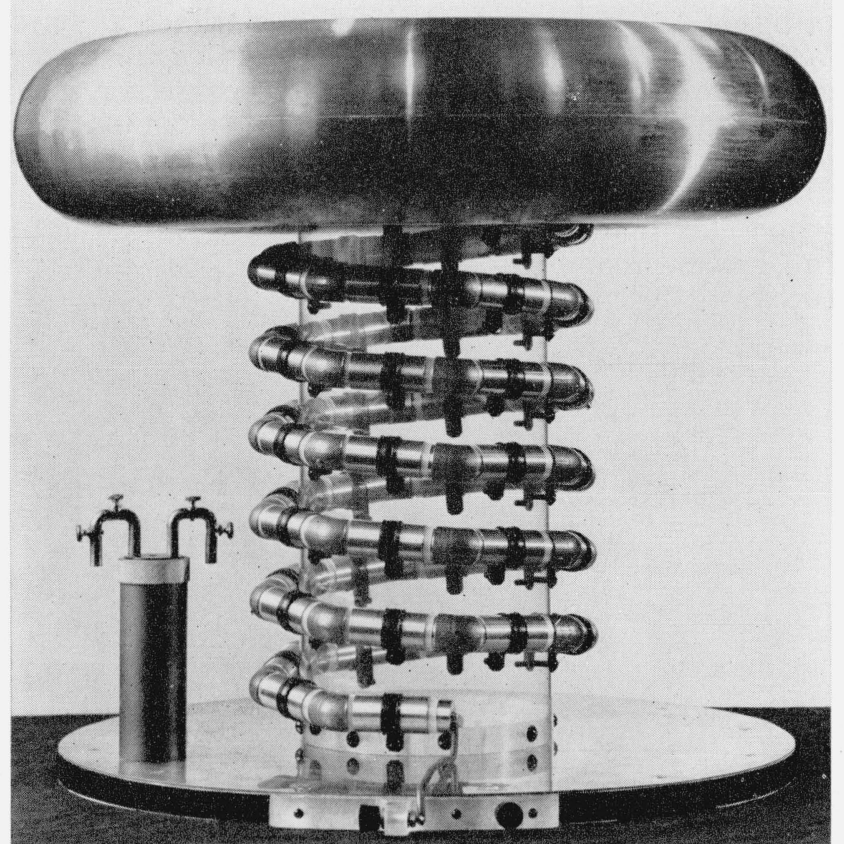

FIgURE 4. 100-megohm, $100 \mathrm{kv}$, helical resistor constructed in 1961 . separate the electrode from the brass plate. Only a visual test could be made; thus the setup was put inside a completely enclosed dark box large enough to house an observer also. With a total voltage of $200 \mathrm{kv}$ between brass plate and electrode there was no visible or audible evidence of corona. Since this was twice the maximum voltage ever to be used with the resistors in place, it was concluded that there would be no corona current off the middle shield electrode when two units are stacked in series. If more than two units are to be stacked, further corona tests should be made and possibly larger shield electrodes than the one shown in figure 4, which is 22 in. in diameter, would be required.

\section{Accuracy Limits on 1961 Model}

Throughout the design and construction of the 1961 model helical resistor, primary consideration was given to eliminating any possible corona or leakage errors. Corona tests were made using voltages much above the expected maximum rating. These precautions by themselves do not eliminate the possibility of corona and leakage errors. The crucial test for detecting such errors is to measure current "in" and current "out" by the method already described and used for the 1955 model.

The accuracy attained was considerably improved over that in the previous tests. In part the higher accuracy was obtained by using somewhat more sensitive galvanometers and reducing the effects of thermal emf's. However, the main improvement was obtained by stabalizing the d-c voltage supply, getting finer control, and using greater patience. Also, by a modified experimental procedure, any constant measurement errors such as might be introduced by the potentiometer, resistance standards, or standard cells were cancelled. This was done by taking current "in" and "out" measurements at low voltage with the 100-megohm resistor shorted, hefore and after each high-voltage check. Then if the potentiometer reading at balance is the same at high voltage as at low voltage with the resistor shorted, no leakage or corona effects are introduced by the high voltage.

Measurements were made with three different values of current (approximately 0.204, 0.509, and 1.019 ma) giving total voltages across the resistor of about $20.4,50.9$, and $101.9 \mathrm{kv}$. The odd values resulted from the use of 5,000-, 2,000-, and 1,000-ohm NBStype resistance standards in conjunction with an unsaturated standard cell for setting current "in." A 5-dial null-type Wolff potentiometer was used to measure the voltage across a similar standard resistor connected between the resistor low-voltage lead and ground, yielding the current "out." The precision obtainable with stable current and fine control was considered to be within $10 \mathrm{ppm}$. The check of current "in" and "out" with the 100-megohm resistor shorted gave values agreeing within $10 \mathrm{ppm}$. With the 100-megohm resistor unshorted, requiring high voltage to balance current "in" and "out", settings on the Wolff potentiometer were the same within $10 \mathrm{ppm}$ as with the 100 -megohm resistor shorted. 
The accuracy of setting the Wolff potentiometer with 20 and $50 \mathrm{kv}$ across the resistor was within 10 ppm, i.e., the same as when the $100-$ megohm resistor was shorted. With $100 \mathrm{kv}$ across the resistor this accuracy was estimated as within $20 \mathrm{ppm}$ - the small increase being due to greater difficulty in holding current nearly constant at the required value.

Current "in" and "out" data were obtained on several different days over a period of 2 months. Room temperature varied only from 24 to $25^{\circ} \mathrm{C}$ and relative humidity from 37 to 50 percent. Most of these data were taken with the brass ground plate connected to ground and tied to the resistor lowvoltage terminal through the standard resistor used in measuring current "out" (because that is the way the resistor will normally be used). As a further check on possible leakage current errors when two or more complete units are connected in series, data were also taken with the brass ground plate connected to the resistor low-voltage lead and insulated from ground. On all of these tests the current "out" was found to be equal to the current "in" to within the accuracy of measurement. This shows that corona and leakage errors for this helical resistor are less than $10 \mathrm{ppm}$ for voltages up to $50 \mathrm{kv}$ and are not greater than $20 \mathrm{ppm}$ for $100 \mathrm{kv}$.

The only other source of error is that due to the overall temperature coefficient of resistance of the $1-\mathrm{meg} o \mathrm{hm}$ wire-wound resistors. The temperature coefficient from 20 to $40{ }^{\circ} \mathrm{C}$ as measured for each 1megohm resistor used in this resistor was less than 2 ppm per degree C. Also resistors with approximately equal positive and negative temperature coefficients were mounted as pairs. The overall temperature coefficient is estimated to be less than 0.4 ppm per degree C. Thus no correction for room temperature under normal laboratory conditions need be applied. Heating of the 1-megohm units due to current at the higher voltage values, however, might cause a slight error because the resistors are totally enclosed and attain higher temperature than they would in a free air space. An estimate of the magnitude of such heating errors was obtained as described in the following two paragraphs.

During the corona tests evidence of heating errors were detected (as already mentioned) when the resistance of the 1/1/2-turn, 24-unit, test sample was measured at various voltages. These 24 units were all purposely chosen to have large temperature coefficients of the same sign so that temperature rise could be estimated from change in resistance. The average coefficient for the 24 units was $-3 \mathrm{ppm}$ per degree C. The total change in resistance, from a measurement at $9 \mathrm{kv}$ taken immediately after putting voltage on, to a measurement at $12 \mathrm{kv}$ after voltage had been on for about $70 \mathrm{~min}$, was $70 \mathrm{ppm}$. This indicates a temperature rise of about $23{ }^{\circ} \mathrm{C}$ with 0.5 $\mathrm{kv}$ applied across each 1-megohm coil. Thus for the complete $100-\mathrm{megohm}$ resistor it would take $50 \mathrm{kv}$ to produce a $23{ }^{\circ} \mathrm{C}$ temperature rise; but since the complete resistor is made up of temperature compensated pairs, the $50 \mathrm{kv}$ would cause a resistance change of less than $10 \mathrm{ppm}$.
Voltages above $12 \mathrm{kv}$ were also applied to the 24 unit special test resistor in order to estimate possible heating errors at higher voltages. After $24 \mathrm{kv}$ had been applied for $2 \mathrm{hrs}$ the shields around the resistor units became noticeable hot to the touch and the total change in resistance was $270 \mathrm{ppm}$. Based on the $-3 \mathrm{ppm}$ temperature coefficient this would mean a $90{ }^{\circ} \mathrm{C}$ temperature rise for the resistance wire. Since the final 100-megohm resistor has an estimated temperature coefficient of $0.4 \mathrm{ppm}$, as compared to $3 \mathrm{ppm}$ for the 24-unit special test resistor, its maximum change with $100 \mathrm{kv}$ applied would be only 36 ppm. However, this voltage does cause considerable heating and should not be held continuously. It was arbitrarily decided that after applying voltages above $70 \mathrm{kv}$ for $30 \mathrm{~min}$ a cooling period of $30 \mathrm{~min}$ should be allowed with no voltage applied.

\section{Calibration of 100-Megohm Resistor}

As explained in the preceding section, it has been experimentally demonstrated that corona and leakage errors for this 100-megohm resistor remain within certain limits up to $100 \mathrm{kv}$. Within these limits then it is permissible to calibrate or measure its resistance at low values of voltage and then use this measured resistance at much higher voltages to compute resistor $I R$ drop.

One method of calibration was to measure each 1-megohm unit individually by placing it in the "unknown" arm of a Wheatstone bridge. All other arms of the bridge were made up of sealed NBS-type standard resistors except for a decade resistor box used for fine control by supplying from 0.00 to 10.00 ohms in $0.01-0 h m$ steps. Values of all resistances used in the bridge, except the 1-megobm unit being tested, were carefully determined by the Resistance and Reactance Section of the National Bureau of Standards. Temperature corrections were applied when necessary. These measurements were made before the complete resistor was assembled. The total resistance was taken to be the sum of the measured values for the 100 units comprising the complete resistor.

A question now arises as to a possible change in effective resistance of an individual unit due to its being mounted inside its shield caps and their being mounted in turn on the lucite tube using nylon clamps and screws. Such a change could be caused by leakage current either via the lucite tube and nylon clamps or via the polyethelene between shield caps. Normally any leakage current via the lucite tube would have been detected during the current "in" and "out" tests but leakage via the polyethelene would not. To check for a possible error due to such leakage, the resistances of several matched pairs of the 1-megohm units were measured after they had been mounted inside their shields and on the lucite tube. In every case the resistance thus measured agreed to within $10 \mathrm{ppm}$ with the sum of the resistance of the individual units as measured before assembly. 
Another method of calibration was used with the complete resistor assembled. This was to make connections at every fifth brass elbow (every 10th resistor unit) so that the 100-megohm standard could be divided into 10 sections of $10-\mathrm{megohm}$ units. These 10 sections were then connected in parallel and measured as a $1-$ megohm resistor. The resistances of all 10 sections in series could then be readily computed from the parallel resistance value, provided the resistance of each section is equal to 10 megohms (within 0.1 percent). Values were obtained in this manner on several different days and at room temperatures from 23 to $26{ }^{\circ} \mathrm{C}$. All such values agreed with that obtained by summing individual resistor measurements to within $15 \mathrm{ppm}$.

\section{Summary}

A special design of high-voltage d-c resistor made up of a large number of individually shielded 1megohm wire-wound resistors connected in series and arranged in helical form, was investigated and found to be suitable for voltages up to $100 \mathrm{kv}$.

Measurements on the first helical resistor built in 1955 indicated negligible leakage and corona errors to within the accuracy of the measurements made at that time. However the heating errors were considerable (0.01 percent per degree C) in this resistor because of the temperature coefficient of the wirewound 1-megohm units.
A second helical resistor, of the same design, was constructed in 1961 using 100 one-megohm, lowtemperature coefficient, wire-wound units. By using matched pairs of these units having temperature coefficients equal in magnitude and opposite in sign, the final $100-$ megohm resistor was estimated to have a temperature coefficient not greater than $0.4 \mathrm{ppm}$ per degree C. Corona and leakage errors for this resistor as determined using improved measurement techniques were found to be less than $10 \mathrm{ppm}$ at $50 \mathrm{kv}$ and less than $20 \mathrm{ppm}$ at $100 \mathrm{kv}$.

This 100-megohm resistor has a special "hat" or high-voltage electrode designed to give uniform gradients from it to ground. This would allow other similar resistors to be stacked on top of it and connected in series for higher voltages. Corona tests on the electrode indicated the feasibility of such "stacking". Exact limits of accuracy attainable at voltages above $100 \mathrm{kv}$ have not yet been determined.

The construction of the two high-voltage resistors described above was carried out almost exclusively by John L. Mills. Harold N. Cones and John L. Mills performed most of the experimental measurements. 